\title{
Desarrollo de la argumentación mediante el fortalecimiento del aprendizaje autorregulado en estudiantes de Derecho en Chile ${ }^{13}$
}

\author{
Sonia Betancourth Zambrano \\ $\mathrm{PhD}$. en Psicología Escolar y Desarrollo \\ Docente de la Universidad de Nariño, Colombia \\ Correo electrónico: sbetan@gmail.com \\ Taeli Gómez Francisco \\ PhD. en Ciencias Filosóficas \\ Docente de la Universidad de Atacama, Chile \\ Correo electrónico: taeli.gomez@uda.cl \\ Alejandra Hormaza Ortega \\ Psicóloga \\ Universidad de Nariño, Colombia \\ Correo electrónico: alejandrahormaza@gmail.com
}

\section{Andrea Martínez Cabrera}

Psicóloga

Universidad de Nariño, Colombia

Correo electrónico: andreamc0117@gmail.com
Recibido: 08/11/2018

Evaluado: $11 / 02 / 2019$

Aceptado: 27/06/2019

\section{Resumen}

La investigación tuvo como objetivo reconocer los efectos del fortalecimiento del aprendizaje autorregulado en la argumentación. La metodología fue cualitativa, con los parámetros de la investigación acción. La unidad de trabajo se conformó por veintisiete estudiantes de la carrera de Derecho de la Universidad de Atacama-Chile. Los resultados muestran que se obtuvieron avances significativos en cuanto a la creación de argumentos y las fases del aprendizaje autorregulado. Se concluye que el aprendizaje autorregulado contribuye al mejoramiento de la argumentación, por lo que es necesario continuar construyendo y aplicando estrategias para su fortalecimiento.

Proceso de aprendizaje, estrategia de enseñanza, rendimiento académico, educación superior.

13 Para citar este artículo: Betancourth, S., Gómez, T., Hormaza, A., \& Martínez, A. (2020). Desarrollo de la argumentación mediante el fortalecimiento del aprendizaje autorregulado en estudiantes de Derecho en Chile. Informes Psicológicos, 20(2), pp. 185-200 http://dx.doi.org/10.18566/infpsic.v20n2a13 


\title{
Development of argumentation by strengthening self-regulated learning in law students in Chile
}

\begin{abstract}
The research aimed to recognize the effects of strengthening self-regulated learning in argumentation. The methodology was qualitative and used the parameters of action research. The work unit was made up of twenty-seven Law students at the University of Atacama-Chile. The results show that significant progress was made regarding the creation of arguments and the phases of self-regulated learning. It is concluded that self-regulated learning contributes to the improvement of argumentation, so it is necessary to continue building and applying strategies for its strengthening.
\end{abstract}

Keywords

Learning process, teaching strategy, academic performance, higher education.

\section{Desenvolvimento da argumentação mediante o fortalecimento da aprendizagem auto-regulada em estudantes de direito no Chile}

\section{Resumo}

A pesquisa teve como objetivo reconhecer os efeitos do fortalecimento da aprendizagem autoregulada na argumentação. A metodologia foi qualitativa, com os parâmetros da pesquisa-ação. A unidade de trabalho esteve composta por 27 estudantes da carreira de direito na Universidade de Atacama-Chile. Os resultados mostram que houve um progresso significativo na criação de argumentos e nas fases do aprendizado auto-regulado. Conclui-se que 0 aprendizado auto-regulado contribui para 0 aprimoramento da argumentação, sendo necessário continuar construindo e aplicando estratégias para seu fortalecimento. 


\section{ntroducción}

La argumentación es un proceso cotidiano que permite a los sujetos posicionarse frente a cualquier tema (Corral \& Piñeyro, 2012), lo que hace difícil analizar por qué su enseñanza en el aula no es considerada como un elemento central; la respuesta se asocia a la lógica de la educación actual, enfocada en brindar conocimientos, más no en formar estudiantes críticos y reflexivos sobre sí mismos y su entorno (Pérez, 2004).

Castro y Sánchez (2013) esbozan la situación que se presenta dentro de las universidades en relación a la argumentación, planteando que los estudiantes no reconocen posiciones alternativas, tampoco cuestionan los postulados de expertos sobre una temática y adoptan una estructura expositiva basada en la descripción de información; igualmente, Aguilar y Fregoso (2013) señalan que los estudiantes de postgrado también tienen dificultades al comprender los argumentos de documentos investigativos, generando complicaciones para construir puntos de vista propios.

Estos hallazgos permiten comprender que las dificultades en argumentación inciden en las problemáticas dentro de la vida académica. En este sentido, la meta que se ha presentado desde hace varios años y que permite solucionar dificultades en el ámbito educativo, ha sido propiciar un ambiente para que los estudiantes sean sus propios maestros, instruyendo sujetos que reflexionen sobre su proceso de aprendizaje (Schunk \& Zimmerman, 1998), mejorando los aspectos metacognitivos, motivacionales y comportamentales (Zimmerman, 2001; 2002).

Desde estas dos visiones, la problemática en la argumentación y la posibilidad de solventar las dificultades a través del aprendizaje autorregulado, resulta importante brindar soluciones previamente investigadas que se puedan aplicar en el aula, con el interés de aportar beneficios a los estudiantes, docentes, instituciones educativas y a la comunidad científica.

En la estructura propuesta por Toulmin, Rieke y Janik (1984), el argumento está compuesto por aserciones, garantías y evidencias; siendo la aserción una afirmación a defender, la evidencia aquella información confiable de la que proviene la premisa y la garantía, el razonamiento que permite relacionar todo el argumento (Rodríguez, 2004).

Jiménez (2010) sostiene que la argumentación desarrolla competencias científicas básicas en los estudiantes, tales como: a) regulación del aprendizaje, b) comunicación lingüística, c) habilidades para ejercer competencias sociales y ciudadanas y d) participación en prácticas grupales que fomentan el debate.

Por su parte, Monterroso (2015) plantea que la argumentación es una de las estrategias que modifica las formas tradicionales de evaluar al estudiante, centrándose en el cumplimiento de metas, el mantenimiento del esfuerzo y los sistemas de control relacionados con procesos de autorregulación 
que sirven para aplicar lo aprendido en el ámbito educativo y en la vida diaria.

En la presente investigación, se asocia la argumentación con el aprendizaje autorregulado, definido por Zimmerman (2000) como una agrupación de habilidades individuales que permiten el manejo de contingencias ambientales e incluyen el sentido de control personal. Desde el enfoque cognitivo social, el modelo propuesto por Zimmerman (2000; 2013) plantea la vinculación del aprendizaje autorregulado con la elección y participación del estudiante en los métodos usados para satisfacer las metas educativas. En este orden de ideas, al realizar una actividad, el estudiante elegirá las estrategias, los resultados y el contexto en función de su proceso; asimismo, la auto-observación le permitirá encaminar su comportamiento hacia la consecución de metas propias. Adicionalmente, Nuñez et al. (citados por Albarracín \& Montoya, 2015) definen el aprendizaje autorregulado como la capacidad de los estudiantes para regular y controlar su proceso de aprendizaje, a través de estrategias cognitivas, metacognitivas y motivacionales que les permiten construir su propio conocimiento.

De acuerdo al estudio realizado por Panadero y Tapia (2014), existen siete teorías principales en relación al aprendizaje autorregulado, estas son: (a) teoría del condicionamiento operante: la motivación para autorregularse depende de la recompensa que se espera obtener como resultado de la actividad; (b) teoría fenomenológica: el comportamiento se autorregula por la necesidad de sentirse competente y se desarrolla en función de los modelos que se imitan; (c) teoría del procesamiento de la información: la autorregulación se produce en función de la información a la que el sujeto presta atención; (d) teoría sociocognitiva: la motivación para autorregularse depende de las metas personales, la percepción de autoeficacia y las expectativas de resultado; (e) teoría de la volición: asume parte de la teoría sociocognitiva y añade la importancia de conservar activo el valor de la meta; (f) teoría vygostkiana: la motivación para autorregularse se genera por la identidad adquirida en la infancia y las interacciones socio-culturales; y (g) teoría constructivista: se centra en el deseo intrínseco de hallar respuesta a las preguntas propias y en el intento de encontrar el equilibrio tras un conflicto cognitivo.

Por otro lado, Puustinen y Pulkkinen (2001) resaltan cuatro modelos con amplia validación empírica sobre el aprendizaje autorregulado, estos son desarrollados por Winne (1995), Borkowski (1996), Pintrich (2000) y Zimmerman (2000). Los modelos mencionados se enfocan en un mismo constructo, por lo tanto, es plausible considerar que existen puntos de encuentro para sintetizar las etapas del aprendizaje autorregulado. Álvarez (2009) desarrolla esta unión determinando cuatro fases fundamentales para comprender el proceso de aprendizaje autorregulado durante la ejecución de una tarea (ver Tabla 1). 
Tabla 1

Fases y componentes del aprendizaje autorregulado (Álvarez, 2009)

\begin{tabular}{|c|c|c|c|}
\hline Fases & & Componentes & Descripción \\
\hline \multirow{6}{*}{$\begin{array}{l}\text { Fase } 1 . \\
\text { Orientación } \\
\text { a la tarea }\end{array}$} & \multirow{3}{*}{$\begin{array}{l}\text { Factores } \\
\text { personales }\end{array}$} & Conocimientos & Aprendizajes previos sobre la tarea. \\
\hline & & Intereses & Inclinaciones por la temática de la tarea. \\
\hline & & Motivaciones & Resultados positivos y/o negativos asociados a la tarea. \\
\hline & \multirow{3}{*}{$\begin{array}{l}\text { Factores } \\
\text { ambientales }\end{array}$} & Demandas de la tarea & Requerimientos específicos de la tarea. \\
\hline & & Recursos personales & Habilidades y capacidades personales para desarrollar la tarea. \\
\hline & & Posibles dificultades & Posibles problemáticas al realizar la tarea. \\
\hline \multirow{2}{*}{$\begin{array}{l}\text { Fase 2. Toma } \\
\text { de decisiones }\end{array}$} & & Metas & Objetivos que el estudiante plantea lograr con la ejecución de la tarea. \\
\hline & & Acciones de solución & $\begin{array}{l}\text { Comportamientos y pensamientos para el cumplimiento exitoso de } \\
\text { la tarea. }\end{array}$ \\
\hline \multirow{2}{*}{$\begin{array}{l}\text { Fase 3. Ejecución } \\
\text { de acciones }\end{array}$} & & Ejecución & Aplicación de las acciones de solución. \\
\hline & & Seguimiento & $\begin{array}{l}\text { Reconocimiento del cumplimiento de las acciones o dificultades, } \\
\text { señalando sus causas. }\end{array}$ \\
\hline $\begin{array}{l}\text { Fase } 4 . \\
\text { Evaluación }\end{array}$ & & Autoevaluación & $\begin{array}{l}\text { Autoevaluación del desempeño, reconociendo el resultado de la } \\
\text { tarea e identificando la necesidad de generar mejores estrategias. }\end{array}$ \\
\hline
\end{tabular}

Fuente: Elaboración Propia

A continuación se presentan algunos hallazgos sobre el estudio de la argumentación y el aprendizaje autorregulado, con el interés de observar la aproximación de otros autores sobre estas variables. En su investigación sobre argumentación y aprendizaje del conocimiento social, Roser (2007) concluye que, al emplear la argumentación en el aula, se produce un progreso en: a) racionalidad: adquisición de conocimientos, mejor posicionamiento y capacidad reflexiva-explicativa; b) tipología discursiva: exposiciones claras y organizadas, aportando justificaciones coherentes, acompañadas de expresiones léxicas adecuadas; y c) compromiso social: propuestas alternativas a los problemas sociales.

Estos resultados no hacen referencia específicamente al objetivo del presente estudio; no obstante, permiten observar que a partir de la argumentación, se producen cambios favorables en las capacidades del sujeto para reflexionar y disponer de su comportamiento, lo cual se relaciona con el aprendizaje autorregulado.

Varios estudios demuestran la influencia positiva de las estrategias de aprendizaje autorregulado a nivel académico, ya que mejoran el rendimiento de los estudiantes, incrementando la motivación y la integración de nuevas metodologías de enseñanza-aprendizaje (De la Fuente, Pichardo, Justicia \& Berbén, 2008; GarcíaRos \& Pérez-González, 2008; Norabuena, 2011).

Medrano y Moreti (2013) investigaron sobre el impacto de un programa de entrenamiento en autorregulación del estudio y habilidades sociales académicas sobre la ansiedad en universitarios. Los hallazgos indican que la intervención fue 
efectiva para lograr una disminución en los niveles de ansiedad ante los exámenes.

Bascón (2014) presenta un estudio que relaciona la autorregulación y la argumentación, que se centra en el discurso argumentativo como una estrategia de autorregulación que permite paliar los conflictos. Los hallazgos se basan en aspectos de diferencias de género y variables personales en cuanto a la argumentación, sin embargo, descuidan la influencia de la autorregulación como aspecto central del estudio.

La argumentación y el aprendizaje autorregulado son variables relevantes para el desarrollo de procesos educativos, sin embargo, la búsqueda de artículos científicos en bases de datos de libre acceso permite observar que en la actualidad no se encuentran estudios que hayan asociado el aprendizaje autorregulado y la argumentación en estudiantes universitarios, produciendo un vacío teórico que requiere ser atendido. Por lo tanto, la investigación tuvo como objetivo reconocer los efectos del fortalecimiento del aprendizaje autorregulado en la argumentación de estudiantes de último año de Derecho de la Universidad de Atacama-Chile.

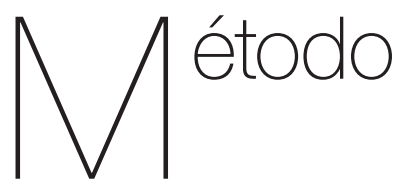

La presente investigación fue cualitativa, desarrollada con los parámetros de la investigación acción como herramienta metodológica para estudiar una realidad, mejorar su comprensión y lograr su transformación (Colmenares \& Piñero, 2008).

\section{Diseño}

Se adaptó el diseño de investigación planteado por Lewin (1946), el cual consta de siete acciones que se sintetizaron en tres momentos:

\section{Primer momento: identificación de la problemática.}

Se especificó la problemática a través de la observación desarrollada por la docente en el transcurso del semestre; para corroborar lo encontrado, se realizó una observación por parte del equipo investigador. A partir de lo anterior, se identificaron dificultades en la argumentación y en el desarrollo del aprendizaje autorregulado, por lo tanto, se consideró relevante fortalecer estas habilidades.

\section{Segundo momento: planificación de acciones.}

Se creó la estrategia compuesta por: (A) tarea de argumentación: creación de un argumento, (B) tarea de aprendizaje autorregulado: descripción del proceso de construcción del argumento, de acuerdo a las fases de aprendizaje autorregulado, y (C) talleres: se desarrollaron dos talleres: 1) importancia del aprendizaje autorregulado, y 2) ficha de aprendizaje autorregulado y fases descritas por Álvarez (2009). Las tareas se desarrollaron en dos ocasiones:

Tarea de argumentación y autorregulación no.1: se realizó para reconocer el proceso de aprendizaje autorregulado 
y el cumplimiento de la estructura del argumento.

Tarea de argumentación y autorregulación no.2: se realizó después de los talleres aplicados, para determinar los cambios en la creación de argumentos y en las fases del aprendizaje autorregulado.

\section{Tercer momento: desarrollo de acciones.}

- Se explicó a los estudiantes la dinámica de las siguientes sesiones y se entregó la tarea de argumentación no.1.

- Los estudiantes entregaron la tarea de argumentación no.1; asimismo, desarrollaron y entregaron la tarea de aprendizaje autorregulado no.1 a la docente.

- Se realizó un taller sobre la importancia de la regulación del aprendizaje en los distintos niveles de educación.

- Se llevó a cabo un taller sobre aprendizaje autorregulado y las fases propuestas por Álvarez (2009). Se entregó a los estudiantes la tarea de argumentación y la tarea de aprendizaje autorregulado no.2; adicionalmente, se brindó información sobre la argumentación y el modelo de Toulmin et al. (1984).

- Los estudiantes entregaron las tareas no.2 y realizaron una exposición reflexiva sobre el cambio entre las tareas no. 1 y no.2.

\section{Unidad de trabajo}

La investigación se desarrolló con estudiantes de la carrera de Derecho de la Universidad de Atacama-Chile. De los 27 estudiantes con los que se trabajó, se analizaron los resultados de 10, entre ellos 7 hombres y 3 mujeres, con edades entre los 22 y los 25 años, quienes cumplieron el total de la actividad entregando las tareas de argumentación y aprendizaje autorregulado no. 1 y no. 2 completas.

\section{Técnicas de recolección de información}

Tarea de argumentación: los estudiantes construyeron individualmente un argumento de acuerdo a una temática entregada por la docente. Se recolectó información sobre la estructura y coherencia del argumento.

Autorreporte sobre el aprendizaje autorregulado: los estudiantes registraron el proceso de desarrollo de la tarea argumentativa mediante un formato con cada una de las fases del aprendizaje autorregulado (ver anexo).

\section{Técnicas de análisis de información}

Se empleó la codificación mediante el proceso propuesto por Ryan y Bernard (2000), analizando las transcripciones de los estudiantes. Se identificaron datos que se clasificaron en categorías establecidas a partir de la revisión teórica: fases del aprendizaje autorregulado y componentes del argumento. 


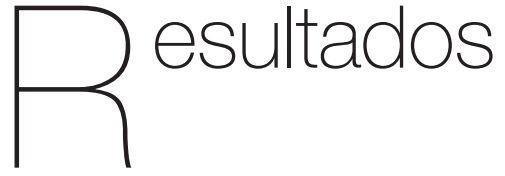

A continuación se presentan los resultados en dos tablas (ver Tabla 2 y 3), en las que se disponen los hallazgos previos y posteriores a la realización de los talleres, adicionalmente, se comentan los logros obtenidos frente a la argumentación y a las fases del aprendizaje autorregulado, al igual que algunos ejemplos sobre el cambio en la argumentación escrita de los participantes.

Tabla 2

Hallazgos de la tarea de argumentación antes y después de la implementación de los talleres.

TAREA DE ARGUMENTACIÓN

\begin{tabular}{|c|c|c|}
\hline Hallazgos previos a los talleres & Hallazgos posteriores a los talleres & LOGROS \\
\hline \multicolumn{3}{|c|}{ 1. Aserción } \\
\hline $\begin{array}{l}\text { Los argumentos presentados suelen iniciar con aspectos } \\
\text { teóricos de otros textos o frases que no suponen una } \\
\text { premisa, lo cual no permite comprender con claridad lo que } \\
\text { se defiende. }\end{array}$ & $\begin{array}{l}\text { Todos los estudiantes comienzan su argumento con una } \\
\text { aserción clara y definida. }\end{array}$ & $\begin{array}{l}\text { Se desarrolló una } \\
\text { mayor apropiación } \\
\text { por parte de los } \\
\text { estudiantes en }\end{array}$ \\
\hline \multicolumn{2}{|c|}{ Ejemplos de los argumentos presentados por los estudiantes } & del argumento, I0 \\
\hline $\begin{array}{l}\text { P1"El derecho a sindicalizarse se encuentra en la } \\
\text { Constitución y dentro del contenido podemos asumir que } \\
\text { se encuentra el derecho a huelga". } \\
\text { P2: "La migración actual en Chile tiene cuatro nociones". }\end{array}$ & $\begin{array}{l}\text { P1: "El paradigma simple otorga una visión reduccionista } \\
\text { a la comprensión de la cultura en el derecho". } \\
\text { P2: "El aporte del paradigma de la complejidad a la } \\
\text { cultura en el derecho es propiciar la diversidad cultural". }\end{array}$ & $\begin{array}{l}\text { coherencia y fuerza } \\
\text { de la postura que } \\
\text { sostenían. }\end{array}$ \\
\hline \multicolumn{3}{|c|}{ 2. Garantías } \\
\hline $\begin{array}{l}\text { Debido a que no existe una aserción clara, las justificaciones } \\
\text { presentadas no pueden comprenderse, puesto que la } \\
\text { relación entre los componentes del argumento influye en la } \\
\text { coherencia del mismo. }\end{array}$ & $\begin{array}{l}\text { Se expusieron varias garantías para sustentar el } \\
\text { argumento, las cuales se encontraban relacionadas con } \\
\text { la aserción planteada y permiten comprender claramente } \\
\text { la premisa a defender. }\end{array}$ & \\
\hline \multicolumn{3}{|c|}{ Ejemplos de los argumentos presentados por los estudiantes } \\
\hline $\begin{array}{l}\text { P1: "El ejercicio de la soberanía reconoce como limitación } \\
\text { el respeto a los derechos esenciales de la naturaleza } \\
\text { humana". } \\
\text { P2: "La perspectiva transnacional de la migración se } \\
\text { entiende como un proceso...". }\end{array}$ & $\begin{array}{l}\text { P1: "...puesto que observa al constructo aislado de su } \\
\text { comprensión total, delimitándola a comportamientos que } \\
\text { caracterizan a un grupo social". } \\
\text { P2: "...esto es debido a que sustituyen los paradigmas } \\
\text { del sistema liberal..." }\end{array}$ & \\
\hline \multicolumn{3}{|c|}{ 3. Evidencias } \\
\hline $\begin{array}{l}\text { En algunos casos no se presentan fuentes o se contemplan } \\
\text { algunas que no cumplen su función dentro del argumento, } \\
\text { ya que no están asociadas con el tema. }\end{array}$ & $\begin{array}{l}\text { Se observa mayor claridad frente a las evidencias; } \\
\text { sin embargo, teniendo en cuenta que se trabajó con } \\
\text { estudiantes de Derecho, se identificaron dificultades } \\
\text { para reconocer evidencias diferentes a la normatividad } \\
\text { jurídica. }\end{array}$ & \\
\hline \multicolumn{3}{|c|}{ Ejemplos de los argumentos presentados por los estudiantes } \\
\hline $\begin{array}{l}\text { P1: "...Según el artículo } 19 \text { de la Constitución Política... } \\
\text { se encuentra como subprincipio la negociación colectiva, } \\
\text { definida por...". } \\
\text { P2: "La migración humana se puede definir como nacional } \\
\text { e internacional". }\end{array}$ & $\begin{array}{l}\text { P1: "Tal como se afirma en el artículo } 111 \text { de la } \\
\text { Constitución Chilena, la cultura... ". } \\
\text { P2: "Bajo esta razón, Prieto (2007) indica que se debe } \\
\text { destacar sin lugar a dudas la importantísima...". }\end{array}$ & \\
\hline
\end{tabular}

Fuente: Elaboración Propia 
Tabla 3

Hallazgos de la tarea de aprendizaje autorregulado antes y después de la implementación de los talleres.

TAREA DE APRENDIZAJE AUTORREGULADO

\begin{tabular}{lll}
\hline Hallazgos previos a los talleres & Hallazgos posteriores a los talleres & LOGROS \\
\hline
\end{tabular}

\section{Fase de orientación a la tarea}

Los estudiantes plantearon que:

1. Sus conocimientos previos sobre el tópico del argumento estaban relacionados con otras asignaturas.

2. El interés por la actividad se relaciona con afinidades por la temática.

3. Los resultados que esperaban lograr con la actividad eran: obtener buenas calificaciones o lograr un mayor aprendizaje sobre la argumentación y la temática del argumento.

4. Existen diversas demandas para la tarea, algunos señalaron que debían crear un caso o consultar sobre una temática.

5. Los recursos que tienen eran las habilidades en la comprensión lectora, sin embargo, la mayoría refirió tener el conocimiento teórico suficiente, lo cual no corresponde a una habilidad sino a un saber previo.

6. Las dificultades son el desconocimiento en la construcción de argumentos, dudas sobre las instrucciones y escaso tiempo.
Los estudiantes señalaron que:

1. Al igual que en la tarea previa a los talleres, sus conocimientos provenían de otras asignaturas.

2. La afinidad con la actividad, al igual que en la anterior tarea, se genera por la materia tratada.

3. Los resultados esperados eran obtener buenas calificaciones y comprender el tópico a profundidad; sin embargo, se adiciona una mayor inclinación por desarrollar un argumento fuerte y sólido, debido a su utilidad en la carrera de Derecho.

4. La demanda de la tarea era construir un argumento de manera adecuada.

5. Sus capacidades son la comprensión lectora y la habilidad para planificar y argumentar.

6. Las dificultades se dan por la escasa práctica en la argumentación.
- Se generó en los estudiantes mayor interés por la argumentación, propiciando reflexiones sobre la influencia de este tipo de habilidades en la carrera de Derecho.

- Se fortaleció en los estudiantes un interés por reconocer adecuadamente los requerimientos de la tarea.

\section{Fase de toma de decisiones}

Los estudiantes mencionan que:

1. Centraron sus metas en mejorar las habilidades argumentativas y obtener calificaciones altas.

2. En el plan de acciones para desarrollar la tarea de argumentación, presentaron dificultades al describir comportamientos concretos y organizados, por ejemplo, quienes sugirieron el uso de horarios, lo hicieron en rangos de tiempo poco específicos.
Los estudiantes plantean que:

1. Las metas frente al ejercicio eran: mejorar las habilidades argumentativas y obtener altas calificaciones.

2. Encontraron dificultad para establecer un plan concreto frente a la tarea. Algunos estudiantes plantearon investigar sobre la temática; sin embargo, no describieron la forma para llevar a cabo esta acción.

\section{FASE 3. Reporte sobre la ejecución y seguimiento del plan determinado}

Los estudiantes describen nuevas acciones para desarrollar la tarea, sin comentar las actividades planteadas en la fase anterior. Algunos señalaron inconvenientes para llevar a cabo lo requerido en esta fase, siendo complejo asociar la elaboración de un plan de acción con la puesta en práctica y el seguimiento del mismo.
Lo encontrado es similar a la tarea desarrollada antes de los talleres, puesto que no existió un plan que permitiera realizar un control; no obstante, algunos estudiantes ejecutaron una búsqueda teórica para desarrollar la tarea; asimismo, comentaron que se presentaron problemas personales que no permitieron ejecutar la actividad adecuadamente.
- No se destaca un cambio significativo en esta fase, ya que se mantienen las ideas fundamentales de la primera tarea.

\section{FASE 4. autoevaluación}

Los estudiantes reportan una percepción positiva sobre el trabajo, planteando que a pesar de presentarse complicaciones, lograron fortalecer sus habilidades argumentativas y ejercer control sobre el aprendizaje, lo que mejoró su rendimiento académico y generó mayor motivación.
Se encontró una percepción positiva frente al desempeño propio; sin embargo, los estudiantes refirieron que las tareas se pudieron ejecutar de mejor manera, pese a esta situación, comprendieron que las acciones realizadas hacen parte de un proceso de mejoramiento continuo.
- Se desarrolló un interés por la búsqueda autónoma de información adicional para la ejecución adecuada de la tarea.

\footnotetext{
Fuente: Elaboración Propia
} 


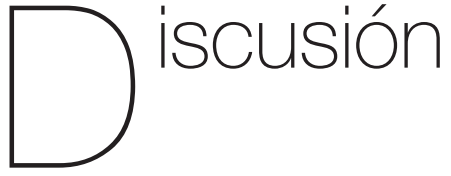

A partir del estudio, se observa que los estudiantes no reconocen el objetivo de la actividad y las metas que el docente planteó al asignar la tarea, siendo elementos fundamentales para el desarrollo del aprendizaje autorregulado. Al respecto, Jorba y Sanmartí (1997) plantean que los universitarios realizan la mayoría de actividades sin identificar cuáles son los objetivos propios y del educador. En este sentido, a través del fortalecimiento del aprendizaje autorregulado, fue posible incentivar el interés por el reconocimiento de los objetivos de la tarea, como se observa en la segunda fase.

Adicionalmente, se evidenciaron dificultades específicas en la argumentación escrita. Bañales, Vega, Reyna, Pérez y Rodríguez (2014) señalan que usualmente los estudiantes tienen problemas en este aspecto debido a: (a) dificultades para comprender el tipo de tarea, (b) problemas para identificar las características de los argumentos y (c) regular la planificación, redacción y revisión para la construcción de un argumento. Al revisar la tarea de aprendizaje autorregulado no.1, se encontró un reporte de cada una de estas problemáticas; sin embargo, en la tarea no. 2 se visualizó un mejor desempeño; este resultado evidencia que al fortalecer las fases del aprendizaje autorregulado es posible mejorar la disposición del estudiante para generar estrategias de aprendizaje propias que permitan alcanzar un progreso en la expresión escrita y argumentativa.
Por otro lado, se encontraron dificultades para añadir evidencias al argumento. Aguilar y Fregoso (2013) brindan una posible aclaración sobre este aspecto, señalando que los estudiantes presentan problemas al emplear datos complejos para explicar un fenómeno, ya que tienen escasas habilidades lectoras, debido a que leen y construyen sus argumentos desde lo explícito, lo cual les resulta fácilmente identificable y comprensible. Es importante aclarar que en la presente investigación se obtuvo un avance con la implementación de los talleres, puesto que los estudiantes agregaron información sobre la normatividad jurídica para justificar sus puntos de vista; no obstante, no se exponen otro tipo de evidencias, como investigaciones científicas, entrevistas, libros, entre otros, aspecto que puede estar relacionado con la formación de los estudiantes en la carrera de Derecho.

Los hallazgos de Fernández et al. (2010) presentan una situación similar a la encontrada en la presente investigación, ya que en la fase de toma de decisiones existe un bajo desempeño en relación con las demás fases del aprendizaje autorregulado. Según estos autores, dicha circunstancia puede suponer un déficit en el establecimiento de objetivos claros, una falta de autoevaluación continua de los progresos o una estimación inapropiada del tiempo necesario para realizar las actividades. Este aspecto es muy importante, ya que la planeación de actividades contribuye a la activación de información que ha sido adquirida previamente, permitiendo la organización y facilitando la comprensión de diversas tareas (Gutiérrez, 2015). Se considera importante reflexionar sobre este aspecto, ya que el aprendizaje autorregulado es un proceso cíclico y las dificultades en alguna de 
sus fases pueden generar problemáticas en las demás.

A través de la investigación, se alcanzaron avances significativos en la creación adecuada de argumentos escritos mediante el fortalecimiento del aprendizaje autorregulado. Esto se relaciona estrechamente con lo expuesto por Álvarez (2012), quien afirma que en un proceso de aprendizaje constante, consciente y autorregulado, los estudiantes se convierten en protagonistas de su propio proceso, establecen distintos puntos de vista, mejoran su capacidad argumentativa y logran fortalecer o cambiar su opinión en relación con una temática particular y, por ende, aprenden a desenvolverse como seres humanos.

Díaz (2002) enfatiza una de las principales razones que motivó el desarrollo de la presente investigación, puesto que plantea su preocupación sobre la dificultad de muchos estudiantes universitarios y profesionales para construir argumentos bien sustentados, coherentes y completos, sin embargo, este autor señala que a partir de metodologías y estrategias novedosas, es posible orientar el desarrollo del aprendizaje autorregulado hacia la solución de esta problemática.

Asimismo, se tuvo en cuenta una de las recomendaciones planteadas en la investigación de Monterroso (2015), quien sostiene que al aplicar estrategias para desarrollar el aprendizaje autorregulado, se deben modificar las formas tradicionales de ejecutar y evaluar este proceso, centrándose en actividades para el cumplimiento de objetivos que estén relacionadas con retos reales para la vida cotidiana; una de estas actividades tiene que ver con la elaboración de argumentos.
A partir de lo comentado, se evidencia que la argumentación escrita, su expresión y potenciación en el aula son elementos básicos para que el estudiante se convierta en un actor clave dentro de su proceso de aprendizaje (SánchezCastaño et al., 2015), puesto que los estudiantes deben aprenden a reflexionar y solucionar sus dificultades a partir de experiencias reales.

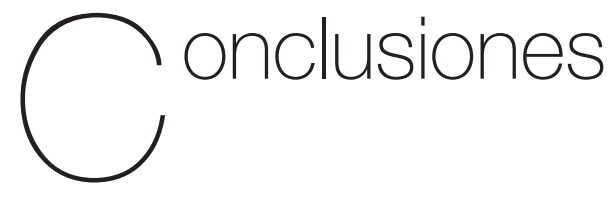

Mediante el desarrollo del proceso de aprendizaje autorregulado, se fortalecieron habilidades argumentativas en los estudiantes, puesto que adquirieron herramientas para construir argumentos sólidos y coherentes a partir del control y monitoreo de acciones. Asimismo, las fases del aprendizaje autorregulado que se fortalecieron a partir de los talleres realizados, son la orientación a la tarea y la autoevaluación.

\section{Aportes}

La ejecución del autorreporte de aprendizaje autorregulado mediante un formato resultó de gran utilidad, ya que permitió un seguimiento del proceso de realización de una tarea, mediante el reconocimiento de las narrativas de los estudiantes. Se concluye que es posible desarrollar este formato con cualquier actividad, por lo tanto, las tareas y talleres propuestos en el presente estudio, pueden ser modificados de acuerdo a las necesidades de los estudiantes 
y a los criterios del docente y de la asignatura.

A partir de la estrategia se identifica la necesidad de desarrollar mayor número de trabajos que traten estas dos variables de manera conjunta, puesto que se han demostrado resultados positivos sobre las habilidades argumentativas de los estudiantes y las fases de autorregulación del aprendizaje, lo cual incidió en resultados académicos satisfactorios; este es un aspecto importante, puesto que la dimensión académica tiende a ser una de las causas principales de deserción en universitarios, convirtiéndose en un aspecto que se debe tener en cuenta tanto por las Instituciones como por el Estado (Ministerio de Educación Nacional, citado por Albarracín y Montoya, 2016).

\section{Limitaciones}

Una de las dificultades presentadas fue la reducida población. Como se comentó anteriormente, se trabajó con estudiantes de último año, por lo que se contó con un número de participantes menor a 30; sumado a esto, algunos estudiantes debían cumplir con deberes adicionales en los tiempos dispuestos para los talleres, lo que ocasionó la disminución de participantes en la investigación.

Por otra parte, se contó con escaso tiempo para desarrollar un mayor número de encuentros, puesto que debían responder a sus actividades académicas dentro y fuera de la Universidad, siendo éste un aspecto por mejorar.

\section{Proyecciones}

Se observa la necesidad de trabajar en todos los niveles educativos con estrategias para comprender la importancia de la autorregulación del aprendizaje y que; a la vez, generen espacios en los que se incentive la aplicación de formatos o estrategias que permitan un registro de sus procesos educativos.

Por otra parte, se podrían desarrollar trabajos en los que se preste atención a la identificación de diferencias poblacionales en relación a la argumentación y al aprendizaje autorregulado, de manera que se puedan fomentar estrategias que optimicen el proceso de aprendizaje en todos los grupos, sin importar sus diferencias.

Por último, se sugiere resolver las dificultades que se tuvo en el presente estudio, aumentando el número de participantes e incrementando la variedad de talleres, con el interés de garantizar mejores resultados.

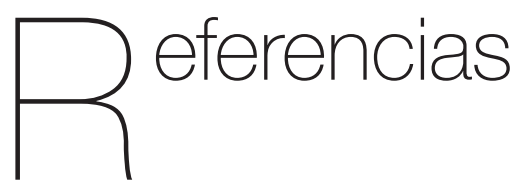

Aguilar, L. \& Fregoso, G. (2013). La lectura de la polifonía e intertextualidad en el texto científico. Revista Mexicana de Investigación Educativa, 18(57), 413-435.

Albarracín, A. \& Montoya, D. (2015). Programas de intervención para estudiantes universitarios con bajo rendimiento académico. Informes Psicológicos, 16(1), 13-43. Recuperado de https://revistas.upb. 
edu.co/index.php/informespsicologicos/ article/view/6470/5940

Álvarez, A. (2012). La autorregulación de los aprendizajes en la asignatura de estudios sociales: el caso del estudiantado de undécimo año del Colegio Bilingüe Santa Cecilia. (Tesis de Maestría). Universidad Estatal a Distancia, Costa Rica.

Álvarez, I. (2009). Evaluar para contribuir a la autorregulación del aprendizaje. Electronic Journal of Research in Educational Psychology, 7(9), 1007-1030.

Bañales, G., Vega, N., Reyna, N., Pérez, A. \& Rodríguez, B. (2014). La enseñanza de la argumentación escrita en la universidad. Revista Mexicana de Investigación Educativa, 20(66), 879-910.

Bascon, M. (2014). Adolescencia, género y conflicto. El discurso argumentativo como herramienta de autorregulación. Boletín de psicología, (110), 7-19.

Betancourt, G. (2018). Influencia de la habilidad argumentativa en el aprendizaje de la reproducción humana en estudiantes de grado octavo. (Tesis de Maestría). Universidad Autónoma de Manizales, Colombia.

Borkowski, J.G. (1996). Metacognition: theory or chapter heading?. Learning and Individual Differences, 8, 391-402.

Castro, M., \& Sánchez, M. (2013). La expresión de opinión en textos académicos escritos por estudiantes universitarios. Revista Mexicana de Investigación Educativa, 18(57), 483-506.

Colmenares, A. \& Piñero, M. (2008). La investigación acción. Una herramienta metodológica heurística para la comprensión y transformación de realidades y prácticas socio-educativas. Laurus, 14(27), 96-114.

Corral, N., \& Piñeyro, N. (2012). Modalidades explicativas y estrategias de justificación. ¿Cómo construyen un punto de vista los estudiantes universitarios de Ciencias de la Educación próximos a egresar?. Educación, Lenguaje y Sociedad, 9(9), 163-197.

De la Fuente, J., Pichardo, M., Justicia, F. \& Berbén, A. (2008). Enfoque de aprendizaje, autorregulación y rendimiento en tres universidades europeas. Psicothema, 20(4), 705-711.

Díaz, A. (2002). La argumentación escrita. Medellín: Universidad de Antioquia.

Fernández, E. et al. (2010). Autorregulación del Aprendizaje en Estudiantes Universitarios. International Journal of Developmental and Educational Psychology, 3(1), 219-225.

García-Ros, R. \& Pérez-González, F. (2008). Validez predictiva e incremental de las habilidades de autorregulación sobre el éxito académico en la universidad, Revista de Psicodidáctica, 16(2), 231-250.

Gutierrez, K. (2015). Perfil agentivo de estudiantes con bajo rendimiento académico: estrategias cognitivas y de control del aprendizaje, autoeficacia académica y motivación. Informes Psicológicos, 15(1), 63-81. http://dx.doi. org/10.18566/infpsicv15n1a04

Jiménez, M. (2010). 10 ideas clave. Competencias en argumentación y uso de pruebas. Barcelona, España: Graó 
Jorba, J. \& Sanmartí, N. (1997). La evaluación como instrumento para mejorar el proceso de aprendizaje de las ciencias. En L. Del Carmen (ed.), La enseñanza y el aprendizaje de las ciencias de la naturaleza en la educación secundaria (pp. 155-199). Barcelona, España: Horsori.

Lewin, K. (1946). Action Research and Minority Problems. Journal of Social Issues, 2, 34-46.

Medrano, L., \& Moretti, L. (2013). Eficacia de un programa de entrenamiento para disminuir manifestaciones cognitivas de ansiedad ante los exámenes en ingresantes universitarios. Informes Psicológicos, 13(1), 41-52. Recuperado de https://revistas.upb. edu.co/index.php/informespsicologicos/ article/view/2342/2086

Monterroso, S. (2015). Aprendizaje autorregulado y su incidencia en el rendimiento académico de los adolescentes. (Tesis de Maestría). Universidad Rafael Landívar. Guatemala.

Norabuena, R. (2011). Relación entre el aprendizaje autorregulado y rendimiento académico en estudiantes de enfermería y obstetricia de la Universidad Nacional "Santiago Antúnez de Mayolo"-Huaraz. (Tesis de Maestría). Universidad Nacional al Mayor de San Marcos. Lima, Perú.

Panadero, E. \& Tapia, J. (2014). Teorías de la autorregulación educativa: una comparación y reflexión teórica. Psicología Educativa, 20(1), 11-22.

Pérez, C. (2004). Cultura, educación y cambio. Revista virtual de la Universidad Católica del Norte, 13. Recuperado de https:// revistavirtual.ucn.edu.co/index.php/ RevistaUCN/article/view/275/520
Pintrich, P. (2000). The role of goal orientation in self-regulated learning. In M. Boekaerts, P.R. Pintrich \& M. Zeidner (eds.), Handbook of Self-regulation. San Diego, CA: Academic Press.

Puustinen, M. \& Pulkkinen, L. (2001). Models of Self-regulated Learning: a review. Scandinavian Journal of Educational Research, 45(3), 269-286.

Rodríguez, L. (2004). El modelo argumentativo de Toulmin en la escritura de artículos de investigación educativa. Revista Digital Universitaria, 5(1), 2-18. Recuperado de: http://www.revista.unam.mx/vol.5/num1/ art2/ene_art2.pdf

Roser, C. (2007). La argumentación en el aprendizaje del conocimiento social. Enseñanza de las Ciencias Sociales, (6), 49-60.

Ryan, G. \& Bernard, H. (2000). Data management and analysis methods. In N.K. Denzin \& Y.S. Lincoln (eds.), Handbook of Qualitative Research, (2a ed.) (pp. 769802). Thousand Oaks, California: Sage.

Sánchez-Castaño, J, Castaño-Mejía, O., \& Tamayo-Alzate, O. (2015). La argumentación metacognitiva en el aula de ciencias. Revista Latinoamericana de Ciencias Sociales, Niñez y Juventud, 13(2), 1153-1168.

Schunk, D. \& Zimmerman, B. (1998). Selfregulated learning: From teaching to selfreflective practice. New York, Estados Unidos: Guilford.

Toulmin, S., Rieke, R. \& Janik, A. (1984). An introduction to reasoning. New York: Macmillan. 
Van Eemeren, F. (2006). Argumentación, comunicación y falacias: una perspectiva pragmadialéctica. Nueva York: Lawrence Erlbaum.

Winne, P. (1995). Inherent details in self autorregulated learning. Educational Psychologist, 30(4), 173-187.

Zimmerman, B. (2000). Attaining self regulation: a social cognitive perspective. In M. Boekaerts \& P. R. Pintrich, (Eds.), Handbook of self-regulation (pp. 13-39). San Diego, California: Academic Press.
Zimmerman, B. (2001). Achieving academic excellence: A self-regulatory perspective. In M. Ferrari (Ed.), The pursuit of excellence through education (pp. 85-110). Mahwah, $\mathrm{NJ}$ : Erlbaum.

Zimmerman, B. (2002). Becoming selfregulated learned: An overview. Theory into Practice, 41, 64-72.

Zimmerman, B. (2013). From cognitivemodeling to self-regulation: a social cognitive career path. Educational Psychologist, 48, 135-147 


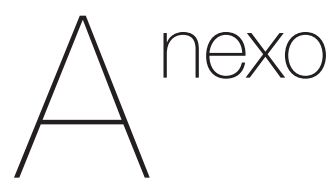

El "Formato de aprendizaje autorregulado" presenta una columna denominada "comentarios", en la cual el estudiante describe su proceso al desarrollar la tarea de argumentación, teniendo en cuenta las fases del aprendizaje autorregulado.

Formato de aprendizaje autorregulado construido por Betancourth, Gómez, Hormaza y Martínez, basado en los planteamientos de Álvarez (2009).

Nombre del Estudiante

Tópico de la argumentación

\begin{tabular}{|c|c|c|c|}
\hline $\begin{array}{l}\text { Fases del aprendizaje } \\
\text { autorregulado }\end{array}$ & Sub fases & Componentes & Comentarios \\
\hline \multirow{6}{*}{$\begin{array}{l}\text { 1.0rientación } \\
\text { a la tarea }\end{array}$} & \multirow{3}{*}{$\begin{array}{l}\text { Factores } \\
\text { personales }\end{array}$} & Conocimientos & \\
\hline & & Intereses & \\
\hline & & Motivaciones & \\
\hline & \multirow{3}{*}{$\begin{array}{l}\text { Factores } \\
\text { situacionales }\end{array}$} & Demandas de la tarea & \\
\hline & & Recursos personales & \\
\hline & & Posibles dificultades & \\
\hline \multirow{2}{*}{ 2. Toma de decisiones } & & Metas & \\
\hline & & Acciones Resolutivas & \\
\hline \multirow{2}{*}{\multicolumn{2}{|c|}{ 3. Ejecución de acciones }} & Ejecución & \\
\hline & & Seguimiento & \\
\hline 4. Evaluación & & Autoevaluación & \\
\hline
\end{tabular}

Observaciones adicionales:

Fuente: Elaboración Propia 